\title{
Risk Evaluation for Personal Identity Management Based on Privacy Attribute Ontology
}

\author{
Mizuho Iwaihara ${ }^{1}$, Kohei Murakami ${ }^{1}$, Gail-Joon Ahn², \\ and Masatoshi Yoshikawa ${ }^{1}$ \\ ${ }^{1}$ Department of Social Informatics, Kyoto University, Japan \\ kmurakami@db.soc.i.kyoto-u.ac.jp, iwaihara@i.kyoto-u.ac.jp, \\ yoshikawali.kyoto-u.ac.jp \\ ${ }^{2}$ Department of Computer Science and Engineering, Arizona State University, USA \\ gahn@asu. edu
}

\begin{abstract}
Identity providers are becoming popular for distributed authentication and distributed identity management. Users' privacy attributes are stored at an identity provider and they are released to a service provider upon user's consent. Since a broad range of privacy information of different sensitiveness can be exchanged in advanced web services, it is necessary to assist users by presenting potential risk on financial and personality damage, before releasing privacy attributes. In this paper, we present a model of privacy attribute ontology and risk evaluation method on this ontology. Then we formalize several matching problems which optimize similarity scores of matching solutions under several different types of risk constraints. We show sophisticated polynomial-time algorithms for solving these optimization problems.
\end{abstract}

\section{Introduction}

A wide variety of new services are created on the web, by connecting existing web services. To carry out services and/or businesses with their customers, many of service providers (SP) require basic personal information of customers, such as name, address, phone number, as well as more critical information such as credit card number. Identity providers (IdPs) offer identity management functionalities, including user authentication and management of basic personal information. Since basic information such as name and email/postal addresses are frequently asked, provisioning of these information from IdP to SP through the user's one-click action can save the user's workload. Liberty Alliance[10], OpenID[11] and CardSpace[1] are proposed identity management standards which provide single sign-on and trust management. However, in these standards, users are still required to carefully examine requested attributes for sensitiveness and criticality. Then users select appropriate identities to be used for the request, where excessive exposure of identities and attributes should be avoided by users' discretion.

Web services are rapidly evolving to cover every kind of social activities among people, and categories of personal attributes are also growing beyond basic attributes. Social network services are offering exchange of very personal attributes such as such as age, ethnicity, religion, height and eye color. For example, orkut(www.orkut.com) 
has an registration form having 30 attributes for "social" page, 16 attributes for "professional" page, and 15 attributes for "personal" page. User-centric control of sharing of personal information is required for healthy support of social activities, and an identity provider of the near future should assist the user through categorization and evaluation of attributes from the point of criticality and sensitiveness.

In this paper, we propose the concept of privacy attribute ontology (PAO), built on the OWL web ontology language[12]. One of primal objectives of PAO is to provide a taxonomy of privacy attributes. Each class of PAO corresponds to a sensitive attribute or an identity, and an individual of the class corresponds to a value of the attribute. IdP manages a PAO as a shared ontology among users as well as a personal information database for each user. Also PAO provides risk evaluation functionality through financial and personality risk values defined on PAO classes. When a service provider presents a list of requested attributes, IdP matches the list with PAO classes, and then the risk values of the requested attributes are evaluated from matched classes. Here we have a number of issues to be solved. First, we need to design a matching algorithm that maximizes linguistic/structural similarities between PAO classes and requested attributes. Secondly, the algorithm also needs to consider risk constraints such that matched classes must not exceed given upper limits of risk values. The algorithm should select a lowrisk combination of identities and attributes associated to these identities, covering requested attributes. In this optimization, we need to consider combination risks which arise if a certain combination of classes is selected for release.

The contribution of this paper is summarized as follows: (1) We present a model of privacy attribute ontology and risk evaluation method on this ontology. (2) We formalize matching problems which optimize similarity scores of matching solutions under three different types of risk constraints. (3) We show sophisticated polynomial-time algorithms for solving the optimization problems of (2).

P3P (Platform for Privacy Preferences Project) [14] is a standard for describing and exchanging privacy policies in XML format. While $\mathrm{P} 3 \mathrm{P}$ is targeted at interpreting privacy practices of service providers, our research is focused on identity providers and users for managing linkages between privacy attributes and identities of different aspects.

Developing ontologies for privacy and trust management on the web has been discussed in the literature [4][5][7]. Our research is different in the way that we focus on risk evaluation for attribute disclosure and selecting disclosing attribute values (individuals) that have minimum risk values. Utilizing semantic web technologies for security and trust management on the web is discussed in [4], which covers authentication, delegation, and access control in a decentralized environment. But an ontology for assessing privacy risk values is not considered.

Matching and aligning ontologies have been extensively studied for integrating ontologies. As a linguistic approach, OntoGenie[13] uses WordNet[16] for extracting ontologies from web pages. Structural similarity is considered in [9] for neural networkbased schema matching. Udrea et al.[15] combined data and structural matching as well as logical inference to improve quality. Our algorithms utilize these linguistic and structural approaches. But we need to deal with the new problem of considering risk values during matching. We have successfully solved ontology matching under various types of risk constraints. 
The rest of the paper is organized as follows. In Section 2 we introduce an existing risk evaluation method for privacy information, and discuss automated risk evaluation based on privacy attribute ontology. In Section 3 , we formalize privacy attribute ontology. In Section 4 we discuss matching requested attributes with PAO classes, and define optimization problems under certain risk constraints. In Section 5, we discuss several issues that need to be solved, and present polynomial-time algorithms for the optimization problems. Section 6 is a conclusion.

\section{Risk Evaluation for Personal Identity Management}

\subsection{JNSA Privacy Risk Evaluation}

Service providers holding customer's privacy data are having risk of privacy leakage. Several measures for evaluating risk of privacy leakage have been proposed. Japan Network Security Association (JNSA) published surveys on information security incidents [6]. The report also presents a method for estimating amount of compensation if a certain portion of privacy data are leaked. The JNSA model is based on classifying reported cases from court decisions and settlements, and the model was validated on these cases. Its evaluation proceeds as follows:

The value of leaked privacy data of an individuation is evaluated in terms of (a) economical loss and (b) emotional pain. The Simple-EP Diagram contains representative privacy attributes according to the dimensions of (a) and (b). Given an attribute, an integer from 1 to 3 is chosen as the value for each dimension. Let $x$ (resp. $y$ ) be the value for (a) economical loss (resp. (b) emotional loss). Then the sensitiveness factor is defined as $E P=\left(10^{x-1}+5^{y-1}\right)$.

Given a collection of privacy attributes for an individual, we take maximum values for $x$ and $y$ from the Simple-EP Diagram. Suppose a record of an individual consists of the attributes: real name, address, birth date, sex, phone, medical diagnosis, bank account and password. Then by the Simple-EP Diagram, the value $(x, y)$ is equal to $(1,1)$ for real name, address, birth date, sex, and phone. On the other hand $(x, y)$ is equal to $(2,1)$ for medical diagnosis, and $(1,3)$ for bank account and password. Since the maximum value for $x$ is 2 and the maximum value for $y$ is 3 , we obtain $E P=35$.

Let the basic information value $B I V$ be 500 points, and let the identifiability factor $I F$ be defined as: $I F=6$ if the individual can be easily identified (for example, real name and address are included), $I F=3$ if the individual can be identified by a certain effort (for example, real name is included, or address and phone are included), and $I F=1$ otherwise (for the case identification is difficult). The leaked privacy information value $L P I V$ is computed by: $L P I V=B I V * E P * I F$. LPIV is designed to approximate the amount of compensation in Japanese yen paid to each leakage victim. The LPIV is further adjusted to reflect other factors such as the social status of the information holder and evaluation on the response after the incident. However, these factors are not directly related to our goal.

The JNSA risk evaluation model can be a basis of risk evaluation for risk-aware identity management, from the points that the model can capture the emotional and financial losses according to a classification of privacy attributes, and it enables quantitative 
comparison of the risks between attributes. However, the method requires human reasoning in determining values from the diagram.

\subsection{Risk Evaluation at Identity Provider}

The basic scenario of personal information management by an identity provider (IdP) utilizing PAO proceeds as follows:

1. IdP manages and holds personal information of the user.

2. The user requests execution of a service to the service provider (SP). SP sends to IdP requested attributes $\mathcal{R} \mathcal{A}$ necessary for the service. $\mathcal{R} \mathcal{A}$ includes basic identity information as well as privacy attributes of the user.

3. IdP matches attributes of $\mathcal{R} \mathcal{A}$ with classes of PAO, to compute releasing classes $\mathcal{R C}$. In the matching process, IdP evaluates risks of releasing information held in $\mathcal{R C}$, and IdP tries to find $\mathcal{R C}$ which has maximum conceptual similarities with $\mathcal{R} \mathcal{A}$, while $\mathcal{R C}$ satisfies a certain risk constraint imposed by the user.

4. $\mathcal{R C}$ is presented to the user. The user modifies and supplements $\mathcal{R C}$ if necessary. Some requested attributes $A$ may not be included in $\mathcal{R C}$, because either $A$ 's risk is intolerable to the user or the user has declined release of of $A$. After SP and the user agree on $\mathcal{R C}$, the information on $\mathcal{R C}$ is sent from IdP to SP.

IdP manages a number of identities of the user, such as student ID, a number of email addresses, citizenship, net identities used for blogs and social network services. Some of these identities are anonymous, while others have solid identities. One identity is associated with a number of attributes, as well as other identities. In selecting $\mathcal{R C}$, IdP needs to find low-risk combination of attributes and avoid linking of identities if it is prohibited by the user.

\subsection{Risk Evaluation Using Privacy Attribute Ontology}

In the following, we summarize the basic notions of our risk evaluation method utilizing PAO.

Risk value is a numerical scale of 1 to 5 representing severity of the risk, where 1 is least severe and 5 is most severe. Risk values are categorized into financial and personality risk values. PAO holds risk values in its classes. However, some classes may not have risk values defined. If a risk value of $C$ is undefined, then the risk value is inherited from $C$ 's super classes. If a class $C$ is in the releasing class $\mathcal{R C}$, then the risk values of $C$ become effective. The risk value of releasing classes $\mathcal{R C}$ is the maximum effective risk value in the classes of $\mathcal{R C}$.

Financial risk value (f-risk value for short) is a risk value for financial damage to the information subject (user). Credit card number, bank account number, and social security number should have high financial risk values. We use $r_{f}(\cdot)$ to denote the financial risk value function on various constructs such as class $C$ and releasing classes $\mathcal{R C}$.

Personality risk value (p-risk value for short) is a risk value for personality damage to the user, including emotional pain, damage to social reputation, and generic damage caused by privacy breach. We use $r_{p}(\cdot)$ to denote the personality risk value function. 
Combined risk value $r_{c}(\mathcal{R C})$ combines f-risk and p-risk values by the function $r_{c}(\mathcal{R C})=\operatorname{cr}\left(r_{f}(\mathcal{R C}), r_{p}(\mathcal{R C})\right)$ such that $c r(x, y)=c_{1} \log \left(\mathcal{F}^{x}+\mathcal{P}^{y}\right)+c_{2}$, where the risk values $x$ and $y$ are converted into an exponential scale by the exponential functions of bases $\mathcal{F}$ and $\mathcal{P}$, and the average of these values are converted back to risk values by the logarithmic function. The bases $\mathcal{F}$ and $\mathcal{P}$ assign weights between the financial and personality risk values, and we can choose $\mathcal{F}=10$ and $\mathcal{P}=5$ following the JNSA model. Constants $c_{1}$ and $c_{2}$ shall be determined to let $\operatorname{cr}(x, y)$ have a range between 1 and 5 .

Combination risk is a risk arising from combination of attributes. Some privacy attributes, such as age and income, may be disclosed under an anonymous username, but combining these attributes with the real name raises the risk of privacy breach. Thus the user should be notified of such high risk combination. Also, the user holding a number of identities at IdP can choose one identity or a combination of identities to cover requested attributes. In this scenario, the user should be advised of the risk in linking several identities. For modeling combination risks, we need to introduce combination risk classes to PAO.

Risk limit is a given upper limit on f-risk, p-risk or combined risk values. If the user gives his/her tolerable risk limit, then disclosing attributes should not exceed the limit. Here exists an optimization problem for finding most-similar matching between the PAO classes and requested attributes, while satisfying the risk limit. Trustability of service providers can be reflected to risk limits, in a way that when dealing with a questionable service provider, the user can define a lower, more cautious risk limit. Detailed linkage between risk limits and existing trustability models is beyond the scope of the paper.

\section{Modeling Privacy Attribute Ontology}

In this section, we formalize privacy attribute ontology. We follow the definitions of OWL[12] as the underlying ontology model. A class represents a concept. A class is associated with zero or more individuals belonging to that class. An ontology can be represented as a directed graph, where nodes are labeled with a class name or an individual, and directed edges are labeled with link types. A link labeled type from an individual to a class represents the membership relation between the individual and the class. A link labeled subclassof from class $C_{1}$ to class $C_{2}$ indicates that $C_{1}$ is a subclass of $C_{2}$ meaning that $C_{1}$ is a concept more specific than $C_{2}$ and an individual belonging to $C_{2}$ also belongs to $C_{1}$. A link labeled partof from class $C_{1}$ to class $C_{2}$ indicates that $C_{2}$ is a composite class composed of a number of component classes, including $C_{1}$. Formally, if a class $C_{1}$ is connected to a class $C_{2}$ through a directed path of partof and subclassof links, then $C_{1}$ is a component class of $C_{2}$. partof links are not allowed to form a directed cycle. We define composite attributes for requested attributes, similarly to composite classes.

PAO has two special link types named $\mathrm{f}$ inancialRisk and personalityRisk, representing the financial risk value $r_{f}(C)$ and and personality risk value $r_{f}(C)$ of a class 


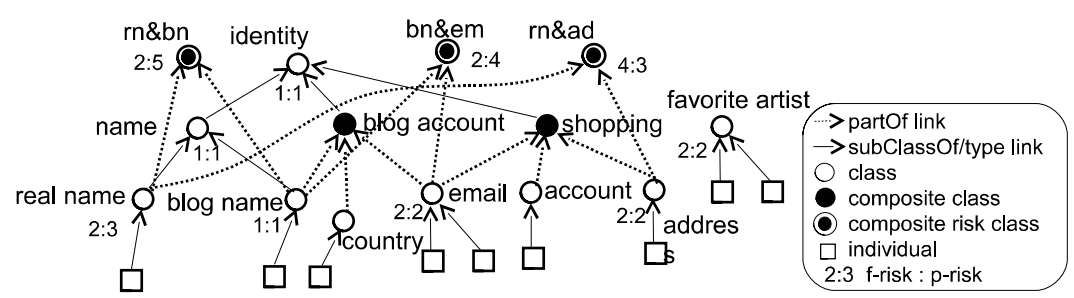

Fig. 1. Privacy attribute ontology

$C$, leading to individuals of real numbers in the range [1.0, 5.0]. An example of privacy attribute ontology is shown in Figure 1, where risk values are shown as numbers of the form $r_{f}: r_{p}$. Also, composite classes are depicted as black circles.

In PAO, we assume that each individual belongs to a single class. For an individual $i$ belonging to multiple classes, we can insert a virtual class between $i$ and these classes, to satisfy the single-class restriction. Thus this is not a tight restriction. Also, if some risk values need to be defined on particular individuals, we create a class for such an individual, and let all the risk values be defined on classes.

As discussed in Section 2.3, we introduce a combination risk class, which is a composite class connected by partof links from its component classes. In Figure 1 combination risk classes are depicted as double circles. The risk value of a combination risk class is applied if all of its component classes are selected for release. For example, the class rn\&bn represents that if real name and blog name are going to be released, then its risk values 2:5 will be applied. These values are higher than that of classes real name and blog name alone, indicating that combination of these classes increase the risk values, or it can be interpreted that the user is not allowing linking of these identities. Thus a combination risk class should have f-risk and p-risk values no less than that of its component classes.

PAO can be shared by a group of users so that the users' common knowledge on risks can be reflected. However, each user may have different views on privacy, and individuals in the ontology are also user-dependent. Thus personalization of PAO is necessary. Personalization of PAO can be done by the following ways: (a) overriding financial and/or personality risk values of a class, (b) adding individuals to a class, and (c) adding a class as a subclass of an existing class. Sharing and personalization of PAO is beyond the scope of this paper, so we do not elaborate on this direction any further.

\section{Matching PAO and Requested Attributes}

\subsection{Matching Problems}

Now we discuss evaluating risk of a set $\mathcal{R} \mathcal{A}$ of requested attributes sent by a service provider, utilizing PAO. Then using the risk evaluation method, we consider optimization problems to find an optimum combination of releasing individuals that achieves given risk constraints.

For associating individuals of PAO and requested attributes $\mathcal{R} \mathcal{A}$, we consider the following two-staged approach: First find a bipartite matching between classes of PAO 
and $\mathcal{R} \mathcal{A}$, then choose an individual from each class selected by the matching. A bipartite matching finds a one-to-one mapping between classes and $\mathcal{R} \mathcal{A}$. Since we assumed that each individual belongs to a single class, this process is straightforward.

We introduce similarity score $\sigma(C, A) \geq 0$ on a PAO class $C$ and a requested attribute $A \in \mathcal{R} \mathcal{A}$. When $\sigma(C, A)>\beta$ holds for a given lower threshold $\beta, C$ and $A$ are regarded as distinct concepts. We discuss construction of $\sigma$ by linguistic similarities in Section 4.2. We construct a matching graph $G_{\sigma, \beta}=(\mathcal{C}, \mathcal{R} \mathcal{A}, E)$ which is a bipartite graph such that $\mathcal{C}$ is the set of classes in PAO, $\mathcal{R} \mathcal{A}$ is the set of requested attributes, and $E$ is the set of edges $\left(C_{i}, A_{j}\right)$ such that $C_{j} \in \mathcal{C}, A_{j} \in \mathcal{R} \mathcal{A}$, and $\sigma(C, A)>\beta$ is true. We also use the similarity function $\sigma$ for edge weights of the bipartite graph $G_{\sigma, \beta}(\mathcal{C}, \mathcal{R} \mathcal{A}, E)$. The weighted bipartite matching problem can be solved in $O\left(N^{3}\right)$ time by the Hungarian method [8], where $N$ is the number of nodes in $G_{\sigma, \beta}$. A matching $M$ on bipartite graph $G_{\sigma, \beta}=(\mathcal{C}, \mathcal{R} \mathcal{A}, E)$ is a bipartite subgraph $\left(\mathcal{C}_{M}, \mathcal{R} \mathcal{A}_{M}, E_{M}\right)$ such that $C C_{M} \subseteq \mathcal{C}, \mathcal{R} \mathcal{A} \subseteq \mathcal{R} \mathcal{A}_{M}, E_{M} \subseteq E$, and no edge in $E_{M}$ conflicts each other, that is, any two edges in $E_{M}$ are not adjacent at either end. Let $\sigma(M)$ denote the sum of the edge weights of $E_{M}$. A matching $M$ on $G_{\sigma, \beta}$ is a maximum matching if $\sigma(M) \geq \sigma\left(M^{\prime}\right)$ holds for any matching $M^{\prime}$ on $G_{\sigma, \beta}$.

Figure 2 shows an example of matching graphs. The nodes on the left are classes of $\mathrm{PAO}$, and the nodes on the right are requested attributes. Here, '+' sign means a composite class or attribute, and '-' sign means a component class or attribute. Edge weights are not displayed in the graph. A matching is shown as bold edges in Figure 2 Notice that this matching includes edges (email, e-mail) and (address, address). These associations may appear reasonable, but unacceptable because structural integrity is ignored. The email class of PAO is a component of class blog account, while address of PAO is a component of class shopping. These composite classes represent distinct identities, and component classes should not be intermixed. Intuitively, a proper matching should preserve component-composite relationships. In Section 5.1 we discuss this component integrity and present a solution. We note that combination risk classes should be excluded from matching candidates, because they are just for internally defining combinational risk values.

Recall that the combined risk value is determined by maximum f-risk value $r_{f}$ and prisk value $r_{p}$ found in releasing classes. In a matching $M=\left(\mathcal{C}_{M}, \mathcal{R} \mathcal{A}_{M}, E_{M}\right)$, the set of matched classes $\mathcal{C}_{M}$ is the releasing classes. Let $r_{f}(M)$ and $r_{p}(M)$ be the maximum f-risk and p-risk values in $M$, respectively. Then the combined risk value $r_{c}(M)$ is computed by $\operatorname{cr}\left(r_{f}(M), r_{p}(M)\right)$.

Requested attributes $\mathcal{R} \mathcal{A}$ may not have any matchable class in $\mathcal{C}$. Such a dangling attribute can be reported by the matching algorithm. In this case, the system needs to start a dialog with the user to create a new class in PAO for the attribute.

By using predefined parameters on risk limits and similarity score limits, a number of optimization problems can be defined:

1. (similarity score maximization) Tolerable upper risk limits $m_{f}$ and $m_{p}$ are given by the user, where $m_{f}>0$ and $m_{p}>0$ are maximum $f$-risk value and maximum $p$-risk value, respectively. The optimization problem is to find a matching $M$ such that similarity score $\sigma(M)$ is maximum and $r_{c}(M) \leq c r\left(m_{f}, m_{p}\right)$ holds. The user may specify $m_{f}=\infty$ and/or $m_{p}=\infty$ if he/she does not restrict one or both of 


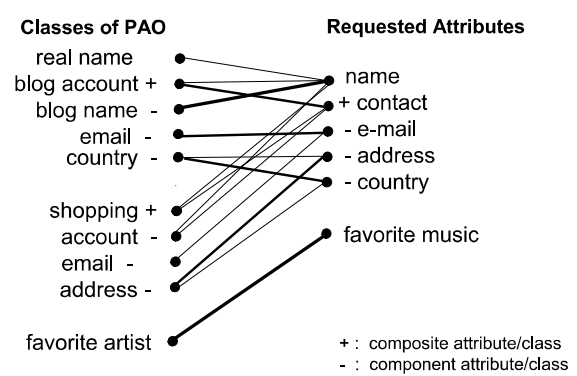

Fig. 2. Matching graph

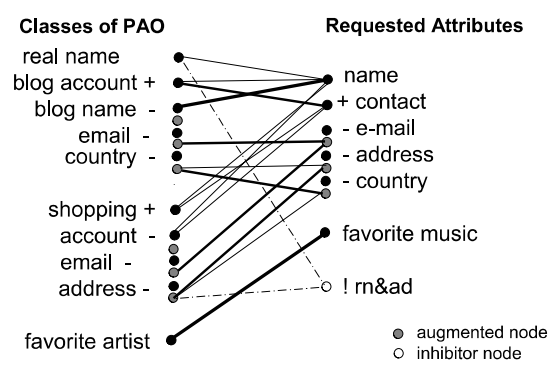

Fig. 3. Augmented matching graph

the risk values. Another version of the problem is similarity score maximization under combined-risk limit $m_{c}$, which is to find a maximum matching under the constraint $r_{c}(M) \leq m_{c}$. In this case, we need to test varying combinations of $m_{f}$ and $m_{p}$ that satisfy $\mathrm{cr}\left(m_{f}, m_{p}\right) \leq m_{c}$.

2. (risk minimization) This problem assumes that a lowerbound $w_{\min }$ for the total similarity score is given. The problem is to find a matching $M$ such that $\sigma(M)>$ $w_{\text {min }}$ and the combined risk value $r_{c}(M)$ is minimum.

3. (combined score maximization) Let $s_{c}(M)$ be combined total score defined by $s_{c}(M)=\sigma(M) / r_{c}(M)$. The problem is to find a matching $M$ that maximizes $s_{c}(M)$. Unlike others, this problem does not have a predefined parameter.

Since specifying all the risk values is tedious, it is likely that some classes or individuals may not be given risk values in PAO. For this issue, we utilize subClassOf links of PAO for inferring risk values, based on the principle that a subclass inherits a missing property value from its parent. Here we also adopt the principle of taking the highest possible risk value, for conservative risk estimation.

(subClassOf rule) Let $C$ be a class in PAO such that $r_{f}(C)$ is undefined. Let $C_{1}, \ldots$, $C_{k}$ be classes in PAO such that there is a path $S_{i}$ of subClassOf links from $C$ to each $C_{i}$ and $C_{i}$ is the only class in $P_{i}$ where $r_{f}\left(C_{i}\right)$ is defined. Then let $r_{f}(C):=$ $\max \left(r_{f}\left(C_{1}\right), \ldots, r_{f}\left(C_{k}\right)\right)$. For the case $r_{p}(C)$ is undefined, simply replace $r_{f}$ with $r_{p}$.

Applying the subClassOf rule to every class that has an undefined risk value gives us a unique PAO that has no undefined risk value, and this procedure can be done in linear time.

\subsection{Linguistic Similarity}

We use Jaro-Winkler score[2] for string similarity and WordNet similarity [16] for score on synonymity. Jaro-Winkler is effective for matching pairs having common substrings, such as between e-mail, email, Email and netmail. WordNet is a lexical dictionary, where words are ground into synonyms (synsets), each synset expressing a distinct concept. WordNet similarity is measured by conceptual-semantic and lexical relations between synsets. We use the sum of Jaro-Winkler score and WordNet score as the similarity score $\sigma$ between PAO classes and $\mathcal{R} \mathcal{A}$. 
Table 1 shows an experimental result on matching names of PAO classes and attributes from web sign-up forms. We constructed a PAO containing 186 classes. The class names of this PAO are matched with two attribute sets from web sign-up forms of eBay and PayPal, using the above similarity score $\sigma$. Case 2 is matches detected by string similarity, which included pair "Primary telephone number" and "Primary telephone". Case 3 is matches detected by synonymity, which included pair "Secret Question" and "Security Question". Overall, the similarity score $\sigma$ is showing enough accuracy for matching requested attributes with PAO.

Table 1. Linguistic matching result on PAO having 186 classes

\begin{tabular}{|c|l|c|c|}
\cline { 2 - 4 } \multicolumn{2}{l|}{} & $\mathrm{eBay}$ & PayPal \\
\hline \hline 1 & string match with PAO classes & 17 & 23 \\
\hline 2 & $\begin{array}{l}\text { attributes matched by string similarity(Jaro- } \\
\text { Winkler) }\end{array}$ & $7 / 3$ & 15 \\
\hline 3 & $\begin{array}{l}\text { attributes matched by synonymity score (Word- } \\
\text { Net) (Excluding case 2) }\end{array}$ & $3 / 5$ & $1 / 2$ \\
\hline 4 & attributes having no matching class in PAO & 2 & 0 \\
\hline \multicolumn{1}{|c|}{ (detected matches)/(correct matches) } \\
\end{tabular}

\section{Matching Algorithms}

\subsection{Component Integrity and Two-Level Matching}

First we formalize component integrity, and present a matching algorithm that achieves a certain type of component integrity while maximizing similarity score. At this moment, we assume that $m_{f}=\infty$ and $m_{p}=\infty$ hold, namely no constraint is given on risk values. We also assume that the PAO has no combination risk class. We extend the algorithm later in this section.

(component integrity) Let us consider a matching $M$ between PAO classes $\mathcal{C}$ and requested attributes $\mathcal{R} \mathcal{A}$. A matching $M$ is said to satisfy component integrity, if the following holds: Let $(C, A)$ and $(D, B)$ be any pair of edges in $M$ such that $C, D \in \mathcal{C}$ and $A, B \in \mathcal{R} \mathcal{A}$. Then $C$ is a component class of $D$ if and only if $A$ is a component attribute of $B$.

Note that PAO can have a multi-level component class, i.e., a composite class can be a component class of another class. PAO can also include a component class shared by multiple composite classes. In such a DAG-structured PAO, imposing the above component integrity becomes a hard problem:

Theorem 1. Given a bipartite graph $G_{\sigma, \beta}=(\mathcal{C}, \mathcal{R} \mathcal{A}, E)$ and a minimum weight $w$, deciding whether $G_{\sigma, \beta}$ has a matching $M$ having weight $\sigma(M)>w$ and satisfying component integrity is NP-complete.

Proof. (sketch) Transformation from SET PACKING[3]. 
Thus it is intractable to enforce the above composite integrity. Also this integrity does not consider link connectivities. In object-oriented modeling, link connectivities are often used to add different perspectives to a class. For example, consider the following subgraphs containing the class email in Figure 1, email $\rightarrow$ blog account, email $\rightarrow$ shopping, and email. Note that the last subgraph is a singleton node. These subgraphs represent e-mail of the blog account, email of the shopping identity, and emails of the person, respectively. Thus each subgraph is representing a different concept.

Now let us consider the following multi-level nesting of composite classes for matching: A class $C$ is a level- $k$ component class of $D$ if (1) for $k=1, C$ is a component class of $D$, and (2) for $k>1, C$ is a component class of a level- $(k-1)$ component class of $D$.

We can adopt the interpretation such that for each different $k$, each path from a composite class to its level- $k$ component class represents a distinct concept. To treat these paths as distinct concepts in matching, new nodes shall be created for each path for varying $k$. However, since PAO can have shared component classes, the number of such paths can be exponential to $k$. Thus considering all the paths to level- $k$ component classes as matching candidates is impractical. In the following, we resrict level $k$ to be 1 , and augment the matching graph $G_{\sigma, \beta}$ with new nodes representing pairs of composite classes and their level-1 component classes. For each composite class $D$ and component class $C$, we create a new node labeled with the concatenation D.C. Likewise, we create a new node labeled with the concatenation $B$.A for composite attribute $B$ and composite attribute $A$. Formally, let $G_{\sigma, \beta}^{a}=\left(\mathcal{C}^{a}, \mathcal{R} \mathcal{A}^{a}, E^{a}\right)$ be the bipartite such that $\mathcal{C}^{a}=\mathcal{C} \cup\{D . C \mid D, C \in \mathcal{C}, C$ is a component class of $D\}, \mathcal{R} \mathcal{A}^{a}=$ $\mathcal{R} \mathcal{A} \cup\{B . A \mid B, A \in \mathcal{R} \mathcal{A}, A$ is a component attribute of $B\}$. The edge set $E^{a}$ is obtained by adding edge $(D . C, B . A)$ to $E$ for each new class D.C and new attribute $B$. A satisfying $\sigma(D . C, B . A)>\beta$, and removing edge $(C, A)$ from $E$ where $C$ and $A$ are component class and attributes, respectively. Here we remove the edge $(C, A)$ because it will be represented by the new component-level edge (D.C, B.A). We call $G_{\sigma, \beta}^{a}$ a composite-augmented graph. Also, we call a bipartite matching $M^{a}$ on $G_{\sigma, \beta}^{a}$ an augmented matching.

For a class $C$ shared by composite classes $D_{1}, \ldots, D_{m}$ in $G_{\sigma, \beta}, G_{\sigma, \beta}^{a}$ has duplicated nodes $C, D_{1} . C, \ldots, D_{m} . C$. Thus an augmented matching $M^{a}$ can include one or more nodes from $C, D_{1} . C, \ldots, D_{m} . C$ in its edges. The following realizes integrity of level-1 component classes in augmented matching:

(augmented component integrity) An augmented matching $M^{a}$ is said to satisfy augmented component integrity, if the following holds: Let (D.C,B.A) and $\left(D_{1}, B_{1}\right)$ be any pair of edges in $M^{a}$ such that $D_{1}, C_{1} \in \mathcal{C}, D . C \in \mathcal{C}^{a}, A, B \in \mathcal{R} \mathcal{A}$, and $B . A \in \mathcal{R} \mathcal{A}^{a}$. Then $D_{1}=D$ holds if and only if $B_{1}=B$ holds.

To satisfy augmented component integrity, we divide the matching of PAO and $\mathcal{R} \mathcal{A}$ into two phases: First, we take each composite class $D$ and each composite attribute $B$ and solve matching between the component classes and attributes of $D$ and $C$, and then augment the (linguistic) similarity score $\sigma(D, B)$ with the matching score (component-level matching). Secondly, we solve matching between the component classes and component attributes using the augmented scores (composite-level matching). The matching algorithm PAOMatch is shown in Figure 4 
1. For each class $D$ in $\mathcal{C}$ and for each attribute $B$ in $\mathcal{R} \mathcal{A}$, compute augmented score $\sigma^{a}(D, B)$ as follows:

1.1 If either $D$ or $B$ is not a composite class/attribute, then let $\sigma^{a}(D, B)=\sigma(D, B)$ and goto Step 1.

1.2 $/ *$ Now $D$ is a composite class and $B$ is a composite attribute. */ Let $C_{i}(i=1, \ldots, k)$ be the component classes of $D$. Let $A_{j}(j=1, \ldots, m)$ be the component attributes of $B$.

1.3 Let $G_{D B}$ be the bipartite graph such that its two node sets are $\left\{D . C_{i}\right\}$ and $\left\{B \cdot A_{j}\right\}$, respectively, and each edge $\left(D . C_{i}, B \cdot A_{j}\right)$ has augmented weight $\sigma^{a}\left(D . C_{i}, B . A_{j}\right)$. If $\sigma^{a}\left(D . C_{i}, B . A_{j}\right)$ is undefined for some $i$ and $j$, then recursively apply Step 1.1-1.4 to obtain $\sigma^{a}\left(D . C_{i}, B . A_{j}\right)$.

1.4 Solve weighted bipartite matching on $G_{D B}$ to obtain matching $M_{D B}$ and its total maximum weight $w_{D B}$. Let $\sigma^{a}(D, B)=\sigma(D, B)+\lambda \cdot w_{D B}$. Here, $0<\lambda<1$ is a pre-defined damping factor.

2. I* Now $\sigma^{a}(D, B)$ is defined for each $D$ and $B$. Note that $G_{\sigma^{a}, \beta}$ does not include augmented nodes. */

Solve weighted bipartite matching on $G_{\sigma^{a}, \beta}$, where edge weight $\sigma$ is replaced by $\sigma^{a}$, and obtain matching $M$.

3. Construct solution matching $M^{a}$ as follows: For each matching edge $(D, B)$ in $M$, add the matching $M_{D B}$ obtained at Step 1.3 to $M$.

Fig. 4. PAOMatch: Two-phased structural matching

Step 1 of PAOMatch computes maximum matching for each component classattribute pair. Then the resulting weight $w_{D B}$ is added to the linguistic similarity score $\sigma(D, B)$, to reflect structural similarity of the components of $D$ and $B$ (Step 1.4). Here, damping factor $0<\lambda<1$ is introduced to reflect the nesting level of component hierarchy. A component class or attribute far from its composite root will have a reduced influence to the score.

After solving maximum matching for each composite class and each composite attribute, the top-level matching is carried out (Step 2). Here, we use $G_{\sigma, \beta}^{a}$ to exclude component classes and component attributes, since component-level matching is already done at Step 1 .

Figure 3 shows application of PAOMatch. Gray nodes are augmented nodes created for each component class/attribute at Step 1.3 of PAOMatch. At Step 1.4, Component-level matching is done between the augmented nodes of composite classes $\{\mathrm{bl}$ log account, shopping $\}$ and attribute $\{$ contact $\}$. Using the scores of these matchings, compositelevel matching is carried out (Step 2). In Figure 3, edge (blog account, contact) is chosen as one of the four composite-level edges. Thus edges (blog account . email, contact.e-mail) and (blog account. country, contact.country) are added at Step 3, as the result of component-level matching. On the hand, although component-level edges (shopping.email, contact.e-mail), (shopping. address, contact.address) are matched at component-level matching, they are eventually discarded because their parents shopping and contact are not matched. Notice that blog name is matched to name at the composite level, not as the composite class blog account. blog name. 
Theorem 2. For a matching graph $G_{\sigma, \beta}=(\mathcal{C}, \mathcal{R} \mathcal{A}, E)$, let $N$ be the number of nodes and $E$ be the number of partof links in $G_{\sigma, \beta}$. Then PAOMatch returns a maximum matching satisfying augmented component integrity in $O\left(N^{3}+E^{3}\right)$ time.

Proof. For augmented component integrity, suppose that augmented matching $M^{a}$ includes edges (D.C, B.A ) and $\left(D_{1}, B_{1}\right)$ such that $D_{1}, C_{1} \in \mathcal{C}, D . C \in \mathcal{C}^{a}, A, B \in \mathcal{R} \mathcal{A}$, and $B . A \in \mathcal{R} \mathcal{A}^{a}$. Now, assume that $D_{1}=D$ holds. Since D.C is an augmented node, the edge (D.C, B.A) must be added at Step 3 of PAOMatch as one of the edge in $M_{D B}$. Since matching at Step 2 guarantees that $\left(D_{1}, B_{1}\right)$ is the only edge in $M^{a}$ that is adjacent to $D_{1}=D$, the composite attribute $B$ of $M_{D B}$ must be $B_{1}$. The only-if part can be shown by a symmetric argument.

For the time bound, first consider Step 1 of PAOMatch. Let $\mathcal{D}$ be the set of composite classes in $\mathcal{C}$, and let $\mathcal{B}$ be the set of composite attributes in $\mathcal{R} \mathcal{A}$. Weighted bipartite matching is executed at Step 1.4 for each $D \in \mathcal{D}$ and for each $B \in \mathcal{B}$. Let $|D|$ (resp. $|B|$ ) denote the number of component classes of $D$ (resp. component attributes of $B$ ). Then one execution of Step 1.4 takes $O\left((|D|+|B|)^{3}\right)$ time. The total time of Step 1.4 is bounded by $\sum_{D \in \mathcal{D}, B \in \mathcal{B}}(|D|+|B|)^{3} \leq\left(\sum_{D \in \mathcal{D}}|D|+\sum_{B \in \mathcal{B}}|B|\right)^{3}=E^{3}$. For Step 2, bipartite matching is performed on $G_{\sigma^{a}, \beta}$, which has $N$ nodes. Thus Step 2 takes $O\left(N^{3}\right)$ time. Step 3 can be done in $O(N+E)$ time.

\subsection{Combination Risk Class and Inhibitor}

Now consider combination risk classes. A combination risk class $D_{r}$ is a composite class having component classes $C_{1}, \ldots, C_{k}$, where $C_{i}$ is a class in $\mathcal{C}$ or componentcomposite classes, and the risk values $r_{f}\left(D_{r}\right)$ and $r_{p}\left(D_{r}\right)$ are given. These risk values are applied when and only when all of $D_{r}$ 's component classes are selected in an augmented matching $M^{a}$. Thus combination risk classes can express high-risk combination of privacy attributes.

Let us consider similarity score maximization where tolerable maximum limits are imposed on f- and/or p-risk values, as we discussed in Section 4.1. If $D_{r}$ exceeds the risk limit, selecting all the component classes of $D_{r}$ should be prohibited in the matching. Now let $\mathcal{D}_{r}$ be the subset of combination risk classes such that $D_{r} \in \mathcal{D}_{r}$ exceeds a given risk limit. We need to design an algorithm that finds a maximum matching that avoids selecting all the component classes for each $D_{r} \in \mathcal{D}_{r}$. To solve this problem, we introduce a combination inhibitor $\operatorname{Inh}\left(D_{r}\right)$, which is a supplementary graph constructed by the algorithm CombInhibitor, shown in Figure 5

Let us reconsider the running example, and assume that p-risk limit $m_{p}=4$ is given. Then rn\&bn is the only combination risk class in Figure 1 that should be inhibited. CombInhibitor adds a component inhibitor for rn\&bn to the augmented matching graph. In Figure 3 the inhibitor node is labeled as ! rn\&ad. The combination inhibitor works as follows: The dashed edges attached to the inhibitor node have the highest weight in the graph. Therefore, if both real name and shopping .address are selected in a matching $M$, we can always make another matching $M^{\prime}$ by replacing one of the matching edges, say, the one adjacent to real name, with (real name, !rn\&ad). Then $M^{\prime}$ should have a score higher than $M$. Therefore maximum matching will give us a solution that avoids simultaneously selecting real name and 
For each combination risk class $D_{r} \in \mathcal{D}_{r}$, do:

1. Add the following bipartite subgraph $\operatorname{Inh}\left(D_{r}\right)=\left(V_{h}, U_{h}, E_{h}\right)$ to the augmented matching graph $G_{\sigma, \beta}^{a}(\mathcal{C}, \mathcal{R} \mathcal{A}, E)$. Let $C_{1}, \ldots, C_{k}$ be the component classes of $D_{r}$.

1.1 The node set $V_{h}$ equals the component classes $\left\{C_{1}, \ldots, C_{k}\right\}$, and the other node set $U_{h}$ equals the singleton set $\left\{A_{h}\right\}$ containing a newly introduced inhibitor node $A_{h}$.

1.2 The edge set $E_{h}$ consists of $k$ edges $\left(C_{1}, A_{h}\right), \ldots,\left(C_{k}, A_{h}\right)$, where each edge has an equal weight $w_{h}$ such that $w_{h}$ is any fixed value higher than the maximum similarity score found in $G_{\sigma, \beta}(\mathcal{C}, \mathcal{R} \mathcal{A}, E)$.

Fig. 5. CombInhibitor $\left(\mathcal{D}_{r}, G_{\sigma, \beta}^{a}\right)$ : Adding combination inhibitors

shopping . addres.s. Thus we have succeeded in preventing p-risk value from exceeding 4. Formally, we have the following property:

Theorem 3. Suppose that a matching graph $G_{\sigma, \beta}=(\mathcal{C}, \mathcal{R} \mathcal{A}, E)$ is augmented with the combination inhibitor Inh $\left(D_{r}\right)$ for each $D_{r} \in \mathcal{D}_{r}$, where $\mathcal{D}_{r}$ is a subset of combination risk classes of $G_{\sigma, \beta}$. Then a maximum matching $M$ of $G_{\sigma, \beta}$ always includes an edge of $\operatorname{Inh}\left(D_{r}\right)$ for any $D_{r} \in \mathcal{D}_{r}$. Thus there is no maximum matching that includes all the component classes of $D_{r}$.

Proof. (omitted due to space limitation)

By the above theorem, just adding combination inhibitor $\operatorname{Inh}\left(D_{r}\right)$ to the matching graph can prevent application of the exceeded risk values of $D_{r}$. The supplementary subgraphs introduced by combination inhibitors have a maximum total size equal to the size of combination risk classes. Thus adding combination inhibitors multiplies the graph size only by a constant factor. We also note that the total maximum weight includes the weight of inhibitor edges given by $w_{i n h}=$ (the number of inhibitors) ${ }^{*} w_{h}$. Thus we need to subtract $w_{i n h}$ from the matching weight $w_{M}$ to obtain the actual total similarity score.

\subsection{Finding Optimum Matching}

We use the following monotonicity in matching solutions for searching on risk values.

Lemma 1. Let $M_{1}^{a}$ be a matching of graph $G_{\sigma, \beta}$ such that $M_{1}^{a}$ satisfies risk limits $r_{f}$ and $r_{p}$. Then there is a matching $M_{2}^{a}$ such that $M_{2}^{a}$ satisfies risk limits $r_{f}^{\prime}>r_{f}$ and $r_{p}^{\prime}>r_{p}$, and $\sigma^{a}\left(M_{1}^{a}\right) \leq \sigma^{a}\left(M_{2}^{a}\right)$.

Proof. It is obvious that $M_{1}^{a}$ remains a matching under the weaker limits of $r_{f}^{\prime}$ and $r_{p}^{\prime}$. Thus at least $M_{1}^{a}$ satisfies the condition of $M_{2}^{a}$ of the lemma.

Let $F$ (resp. $P$ ) be the number of distinct f-risk (resp. p-risk) values appearing in $G_{\sigma, \beta}^{a}$. If we are using 5-digit risk values, then we have $F \leq 5$ and $P \leq 5$. Figure 6 shows matching algorithms for the optimization problems defined in Section 4.1 


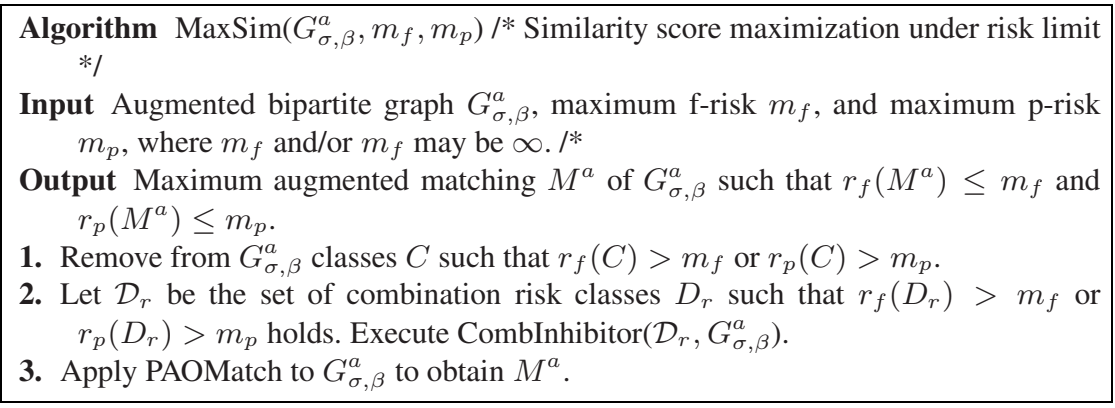

Algorithm MaxSimCombinedRisk $\left(G_{\sigma, \beta}^{a}, m_{c}\right) / *$ Similarity score maximization under combined-risk limit */

Input: Augmented bipartite graph $G_{\sigma, \beta}^{a}$, and maximum combined-risk $m_{c}$.

Output: Maximum augmented matching $M^{a}$ of $G_{\sigma, \beta}^{a}$ such that $\operatorname{cr}\left(r_{f}\left(M^{a}\right), r_{p}\left(M^{a}\right)\right) \leq m_{c}$.

1. Assume that $F<P$. Otherwise in the following steps swap $F$ with $P$, and swap $m_{f}$ with $m_{p}$.

2. For each value $r_{f}$ in $F$ do:

2.1 Compute maximum $r_{p}$ such that $c r\left(r_{f}, r_{p}\right) \leq m_{c}$ holds.

2.2 Call $\operatorname{Max} \operatorname{Sim}\left(G_{\sigma, \beta}^{a}, r_{f}, r_{p}\right)$.

3. Report matching $M^{a}$ that had maximum score at 2.2

Algorithm $\operatorname{MinRisk}\left(G_{\sigma, \beta}^{a}, w_{\text {min }}\right) / *$ Risk minimization under minimum similarity score $w_{\min } *$ /

1. Assume that $F<P$. Otherwise in the following steps swap $F$ with $P$, and swap $m_{f}$ with $m_{p}$.

2. For each value $r_{f}$ in $F$ do:

2.1 Perform binary search on p-values to find minimum $r_{p}$ such that result $M^{a}$ of $\operatorname{Max} \operatorname{Sim}\left(G_{\sigma, \beta}^{a}, r_{f}, r_{p}\right)$ has score no smaller than $w_{\text {min }}$.

3. Report the matching found in Step 2.1 such that its combined risk $r$ is minimum.

Algorithm MaxCombined $\left(G_{\sigma, \beta}^{a}\right) / *$ Combined score maximization. */

1. For each f-risk value $p_{f}$ and for each p-risk value $p_{f}$, do:

1.1 Call $\operatorname{Max} \operatorname{Sim}\left(G_{\sigma, \beta}^{a}, r_{f}, r_{p}\right)$.

2. Report matching $M^{a}$ that had maximum combined score $\sigma\left(M^{a}\right) / r_{c}\left(M^{a}\right)$ in Step 1.1.

Fig. 6. Algorithms for maximum matching under given risk constraints

Theorem 4. Let $F$ (resp. $P$ ) be the number of distinct f-risk (resp. p-risk) values appearing in augmented matching graph $G_{\sigma, \beta}^{a}$. Let $R$ be $F+P$, and let $N$ be the number of nodes and $E$ be the number of partof links in $G_{\sigma, \beta}^{a}$. The following holds:

1. $\operatorname{Max} \operatorname{Sim}\left(G_{\sigma, \beta}^{a}, m_{f}, m_{p}\right)$ solves similarity score maximization in $O\left(N^{3}+E^{3}\right)$ time.

2. MaxSimCombinedRisk $\left(G_{\sigma, \beta}^{a}, m_{c}\right)$ solves similarity score maximization under combined-risk limit $m_{c}$ in $O\left(\left(N^{3}+E^{3}\right) R\right)$ time.

3. $\operatorname{MinRisk}\left(G_{\sigma, \beta}^{a}, w_{\text {min }}\right)$ solves risk minimization under minimum similarity score $w_{\text {min }}$ in $O\left(\left(N^{3}+E^{3}\right) R \log R\right)$ time. 
4. MaxCombined $\left(G_{\sigma, \beta}^{a}\right)$ solves combined score maximization in $O\left(\left(N^{3}+E^{3}\right) R^{2}\right)$ time.

Proof. 1. In Step 1 of $\operatorname{Max} \operatorname{Sim}\left(G_{\sigma, \beta}^{a}, m_{f}, m_{p}\right)$, classes $C$ that violate the maximum limit $m_{f}$ or $m_{p}$ are removed. If these classes $C$ are not removed, it is easy to construct a graph that has a maximum matching violating one of these limits. In Step 2, CombInhibitor introduces combination inhibitors so that by Theorem 3 , any matching of $G_{\sigma, \beta}^{a}$ will not include a combination risk class that violates the limits. If CombInhibitor is not applied, it is easy to construct a graph that has a maximum matching that includes all the component classes of a combination risk class which violates the limits. Thus the matching obtained at Step 3 gives maximum score under the limits $m_{f}$ and $m_{p}$. For the time bound, Step 1 and Step 2 can be done in linear time and increase the size of $G_{\sigma, \beta}^{a}$ by a factor of a constant. Thus by Theorem 2 , Step 3 can be done in $O\left(N^{3}+E^{3}\right)$ time. 2. For similarity score maximization under combined-risk limit $m_{c}$, testing maximum matching score among every combination of risk values $r_{f}$ and $r_{p}$ that satisfy the limit $m_{c}$ guarantees that there will be no other matching that has a higher score while satisfying $m_{c}$. We do not need to test on combinations $r_{f}^{\prime}$ and $r_{p}^{\prime}$ which have combined risk values less than $m_{c}$, since by Lemma matching score satisfying $r_{f}^{\prime}$ and $r_{p}^{\prime}$ does not exceed the score satisfying $r_{f} \geq r_{f}^{\prime}$ and $r_{p} \geq r_{p}^{\prime}$ such that $\operatorname{cr}\left(r_{f}, r_{p}\right) \leq \operatorname{cr}\left(r_{f}, r_{p}\right) \leq m_{c}$. For the time bound, $\operatorname{Max} \operatorname{Sim}\left(G_{\sigma, \beta}^{a}, r_{f}, r_{p}\right)$ is called $F \leq R$ times, which gives the bound $O\left(\left(N^{3}+E^{3}\right) R\right)$.

3. For risk minimization under minimum similarity score $w_{m i n}$, it is sufficient to test all the combinations of f-risk and p-risk values that have matching $M^{a}$ such that $\sigma^{a}\left(M^{a}\right) \geq$ $w_{\text {min }}$ holds. Again by Lemma 1 if a combination of $r_{f}$ and $r_{p}$ has a matching score greater than the minimum limit $w_{\text {min }}$, then all the combinations such that $r_{f}^{\prime} \geq r_{f}$ and $r_{p}^{\prime} \geq r_{p}$ also have matching score greater than $w_{\min }$. This property allows us to perform binary search on $r_{f}$ for each fixed $r_{p}$. Thus $\operatorname{Max} \operatorname{Sim}\left(G_{\sigma, \beta}^{a}, r_{f}, r_{p}\right)$ is called $O(R \log R)$ at Step 2.2 and we have the time bound.

4. For combined score maximization, again it is sufficient to test all the combinations of f-risk and p-risk values to find a matching $M^{a}$ having maximum combined score $s_{c}\left(M^{a}\right)=\sigma\left(M^{a}\right) / r_{c}\left(M^{a}\right)$. Since the combined score $s_{c}=w / r$ does not have monotonicity, we try $\operatorname{Max} \operatorname{Sim}\left(G_{\sigma, \beta}^{a}, r_{f}, r_{p}\right)$ for $O\left(R^{2}\right)$ times.

\section{Conclusion}

In this paper, we proposed the concept of privacy attribute ontology for identity management involving complex attributes and identities. Our ontology model realizes risk evaluation of matching attributes, and the algorithms presented in this paper solve maximum similarity matching under various types of risk constraints.

\section{Acknowledgment}

This work is in part supported by the Grant-in-Aid for Scientific Research of JSPS (Japan Society for the Promotion of Science) (\#18300031), and Strategic International Cooperative Program of JST (Japan Science and Technology Agency). The work of 
Gail-J. Ahn was partially supported by the grants from US National Science Foundation (NSF-IIS-0242393) and the US Department of Energy Early Career Principal Investigator Award (DE-FG02-03ER25565).

\section{References}

1. Microsoft Developer Network (MSDN) CardSpace page, http://msdn.microsoft.com/CardSpace

2. Cohen, W., Ravikumar, P., Feinberg, S.: A Comparison of String Metrics for Matching Names and Records. In: Proc. KDD Workshop on Data Cleaning and Object Consolidation (2003)

3. Garey, M.R., Johnson, D.S.: Computers and Intractability - A Guide to the Theory of NPCompleteness. Freeman, New York (1979)

4. Kagal, L., Finin, T.W., Joshi, A.: A Policy Based Approach to Security for the Semantic Web. In: Fensel, D., Sycara, K.P., Mylopoulos, J. (eds.) ISWC 2003. LNCS, vol. 2870, pp. 402-418. Springer, Heidelberg (2003)

5. Jutla, D.N., Bodorik, P.: Sociotechnical Architecture for Online Privacy. IEEE Security \& Privacy 3(2), 29-39 (2005)

6. Japan Network Security Association, Surveys on Information Security Incidents (in Japanese) (2006), http://www.jnsa.org/result/2006/pol/insident/070720/

7. Kolari, P., Li Ding, S., Ganjugunte, L., Kagal, A.J., Finin, T.: Enhancing Web Privacy Protection through Declarative Policies. In: Proc. IEEE Workshop on Policy for Distributed Systems and Networks(POLICY 2005) (June 2005)

8. Kuhn, H.W.: The Hungarian Method for the Assignment Problem. Naval Research Logistics Quarterly 2, 83-97 (1955)

9. Li, W.-S., Clifton, C.: SEMINT: a Tool for Identifying Attribute Correspondences in Heterogeneous Database Using Neural Networks. Data Knowledge Eng. 33(1), 49-84 (2000)

10. Liberty Alliance Project Homepage, http://www.projectliberty.org/

11. OpenID Foundation, http://openid.net/

12. OWL Web Ontology Language Overview, W3C Recommendation 10 (February 2004), http: //www.w3.org/TR/owl-features/

13. Patel, C., Supekar, K., Lee, Y.: OntoGenie: Extracting Ontlogy Instances from WWW. In: Proc. Huaman Language Technology for the Semantic Web and Web Services, ISWC 2003 (2003)

14. The Platform for Privacy Preferences 1.1 (P3P1.1) Specification, W3C Working Group Note (November 13, 2006)

15. Udrea, O., Getoor, L., Miller, R.J.: Leveraging Data and Structure in Ontology Integration. In: Proc. ACM SIGMOD 2007, pp. 449-460 (2007)

16. WordNet - a Lexical Database for the English Language, Princeton University, http://wordnet.princeton.edu/ 